\title{
Long-term outcomes from the phase II L-MIND study of tafasitamab (MOR208) plus lenalidomide in patients with relapsed or refractory diffuse large B-cell lymphoma
}

Johannes Duell, ${ }^{1}$ Kami J. Maddocks, ${ }^{2}$ Eva González-Barca, ${ }^{3}$ Wojciech Jurczak, ${ }^{4}$ Anna Marina Liberati, ${ }^{5}$ Sven de Vos, ${ }^{6}$ Zsolt Nagy, ${ }^{7}$ Aleš Obr, ${ }^{8}$ Gianluca Gaidano, ${ }^{9}$ Pau Abrisqueta, ${ }^{10}$ Nagesh Kalakonda, ${ }^{11}$ Marc André, ${ }^{12}$ Martin Dreyling, ${ }^{13}$ Tobias Menne, ${ }^{14}$ Olivier Tournilhac, ${ }^{15}$ Marinela Augustin, ${ }^{16}$ Andreas Rosenwald, ${ }^{17}$ Maren Dirnberger-Hertweck,${ }^{18}$ Johannes Weirather, ${ }^{18}$ Sumeet Ambarkhane ${ }^{18}$ and Gilles Salles ${ }^{19^{\circ}}$

${ }^{1}$ Medizinische Klinik und Poliklinik II, Universitätsklinik Würzburg, Würzburg, Germany; ${ }^{2}$ Department of Internal Medicine, Arthur G James Comprehensive Cancer Center, Ohio State University Wexner Medical Center, Columbus, OH, USA; ${ }^{3}$ Department of Hematology, Institut Catalá d'Oncologia (ICO), Hospital Duran i Reynals, Universitat de Barcelona, Barcelona, Spain; ${ }^{4}$ Maria Sklodowska-Curie National Research Institute of Oncology, Kraków, Poland; ${ }^{5}$ Università degli Studi di Perugia, Azienda Ospedaliera Santa Maria di Terni, Terni, Italy; ${ }^{6}$ Department of Medicine, Ronald Reagan UCLA Medical Center, Santa Monica, CA, USA; ${ }^{7} 1^{\text {st }}$ Department of Internal Medicine, Semmelweis University, Budapest, Hungary; ${ }^{8}$ Department of Hemato-Oncology, Palacký University and University Hospital, Olomouc, Czech Republic; 'Division of Hematology, Department of Translational Medicine, University of Eastern Piedmont, Novara, Italy; ${ }^{10}$ Department of Hematology, Vall d'Hebron Institute of Oncology (VHIO), Vall d'Hebron University Hospital, Barcelona, Spain; ${ }^{11}$ Molecular and Clinical Cancer Medicine, University of Liverpool and The Clatterbridge Cancer Centre, Liverpool, UK; ${ }^{12}$ Department of Haematology, Université Catholique de Louvain, CHU UCL Namur, Yvoir, Belgium; ${ }^{13}$ Department of Medicine III, LMU University Hospital, Munich, Germany; ${ }^{14}$ Department of Haematology, Freeman Hospital, Newcastle Upon Tyne Hospitals NHS Foundation Trust, Newcastle Upon Tyne, UK; ${ }^{15}$ Service d'Hématologie Clinique et de Thérapie Cellulaire, CHU Estaing, Clermont-Ferrand, France; ${ }^{16}$ Department of Hematology and Oncology, Paracelcus Medical University, Klinikum Nürnberg, Nürnberg, Germany; ${ }^{17}$ Institute of Pathology, University of Würzburg, Würzburg, Germany; ${ }^{18}$ MorphoSys AG, Planegg, Germany and ${ }^{19}$ Hématologie, Hospices Civils de Lyon and Université de Lyon, Lyon, France.

${ }^{\circ}$ Current address: Lymphoma Service, Memorial Sloan Kettering Cancer Center, New York, NY, USA.

doi:10.3324/haematol.2021.279802

(C)2021 Ferrata Storti Foundation

An error in the original manuscript (2020.275958) is present in the paper that appeared as Early view on July 1 , 2021.

In Table 2, 'Efficacy outcomes in primary and follow-up analysis' The complete response for 'Last therapy refractory' should be 14 (40.0) and not 14 (14.0).

Correction

The corrected datum, a complete response rate of $40.0 \%$ in last-therapy-refractory patients receiving tafasitamab plus lenalidomide for relapsed/refractory diffuse large B-cell lymphoma, is shown in the Table below.

Table 2. Efficacy outcomes in the primary and follow-up analyses.

\begin{tabular}{|c|c|c|c|c|c|}
\hline & \multicolumn{2}{|c|}{ Tafasitamab plus lenalidomide $(\mathrm{N}=80)^{\ddagger}$} & \multicolumn{3}{|c|}{ Clinically relevant subgroups (follow-up analysis) } \\
\hline & $\begin{array}{l}\text { Primary analysis } \\
\text { (data cut-off: } \\
\text { Nov 30, 2018) }\end{array}$ & $\begin{array}{l}\text { Follow-up analysis } \\
\text { (data cut-off: } \\
\text { Oct 30, 2020) }\end{array}$ & $\begin{array}{l}\text { Primary refractory } \\
\text { disease } \\
(n=15)\end{array}$ & $\begin{array}{c}\text { Rituximab-refractory } \\
\text { disease } \\
(n=33)\end{array}$ & $\begin{array}{l}\text { Last-therapy- } \\
\text { refractory } \\
(n=35)\end{array}$ \\
\hline \multicolumn{6}{|l|}{ Best objective response, n (\%) } \\
\hline Complete response & $34(42.5)$ & $32(40.0)$ & $5(33.3)$ & $13(39.4)$ & $14(40.0)$ \\
\hline Partial response & $14(17.5)$ & $14(17.5)$ & $3(20.0)$ & $5(15.2)$ & $7(20.0)$ \\
\hline Stable disease & $11(13.8)$ & $13(16.3)$ & $2(13.3)$ & $4(12.1)$ & $3(8.6)$ \\
\hline Progressive disease & $13(16.3)$ & $13(16.3)$ & $3(20.0)$ & $7(21.2)$ & $7(20.0)$ \\
\hline Not evaluable* & $8(10.0)$ & $8(10.0)$ & $2(13.3)$ & $4(12.1)$ & $4(11.4)$ \\
\hline ORR (CR + PR), n (\%) $[95 \% \mathrm{CI}]^{\dagger}$ & $\begin{array}{c}48(60.0) \\
{[48.4-70.9]}\end{array}$ & $\begin{array}{c}46(57.5) \\
{[45.9-68.5]}\end{array}$ & $\begin{array}{c}8(53.3) \\
{[26.6-78.7]}\end{array}$ & $\begin{array}{c}18(54.5) \\
{[36.4-71.9]}\end{array}$ & $\begin{array}{c}21(60.0) \\
{[42.1-76.1]}\end{array}$ \\
\hline Median DoR (IRC), months (95\% CI) & 21.7 (21.7-NR) & 43.9 (26.1-NR) & NR (1.8-NR) & NR (5.8-NR) & NR (5.8-NR) \\
\hline Median PFS (IRC), months (95\% CI) & 12.1 (5.7-NR) & $11.6(6.3-45.7)$ & 5.3 (0.9-NR) & 7.6 (2.7-NR) & 7.6 (2.7-NR) \\
\hline Median OS, months $(95 \% \mathrm{CI})$ & NR (18.3-NR) & 33.5 (18.3-NR) & 13.8 (1.3-NR) & 15.5 (8.6-NR) & 15.5 (8.6-NR) \\
\hline
\end{tabular}

*Non-evaluable patients had no valid post-baseline response assessments. 'Using the two-sided $95 \%$ Clopper-Pearson exact method based on a binomial distribution. ${ }^{\ddagger}$ One patient received tafasitamab only. ORR: objective response rate; CR: complete response; PR: partial response; 95\% CI: $95 \%$ confidence interval; DoR: duration of response; IRC: independent review committee; PFS: progression-free survival; OS: overall survival; NR: not reached. 